\title{
Adaptive Prediction of Channels with Sparse Features in OFDM Systems
}

\author{
Changwei Lv, Shujuan Hou, and Wenbo Mei \\ School of Information and Electronics, Beijing Institute of Technology, Beijing 100081, China \\ Correspondence should be addressed to Shujuan Hou; shujuanhou@bit.edu.cn
}

Received 30 January 2013; Revised 21 March 2013; Accepted 17 April 2013

Academic Editor: Alfonso Muñoz-Acevedo

Copyright (C) 2013 Changwei Lv et al. This is an open access article distributed under the Creative Commons Attribution License, which permits unrestricted use, distribution, and reproduction in any medium, provided the original work is properly cited.

\begin{abstract}
A time domain channel prediction method exploiting features of sparse channel is proposed for orthogonal frequency division multiplexing (OFDM) systems. The proposed predictor operates in the time domain on each channel tap and separates the negligible taps from significant channel taps before performing prediction. We also compare the proposed prediction method with the classical frequency domain method realized at each OFDM subcarrier and demonstrate that our method increases the prediction accuracy and reduces the computational complexity. Simulations on the physical channel model verify the performance of the proposed method.
\end{abstract}

\section{Introduction}

OFDM has received considerable interest for its advantages of converting frequency-selective fading channels into a parallel collection of frequency flat subchannels and reducing intersymbol interference (ISI) [1]. Adaptive transmission is a promising technique to provide a good throughput performance for OFDM systems [2]. In adaptive transmission, accurate channel state information (CSI) for the upcoming transmission frame is needed. In fast variation channels, the estimated CSI will be soon outdated, and channel prediction is the sole way to provide the future CSI.

Due to the parallel transmission scheme of OFDM systems, existing channel predictors are often realized at each OFDM subcarrier in the frequency domain [3]. The variation at each subcarrier is a combined variation of multiple taps, which makes it difficult to accurately predict the channel. In the time domain, some effective channel predictors (such as [4]) have been proposed. In practical terms, wireless channels are often sparse [5]; that is, there are not so many channel taps with significant energy (if compared to the number of subchannels of OFDM), and several approaches have been proposed to exploit this property in channel estimation. One of the most widely used approaches is significant tap selection method $[5,6]$, which uses a measure to determine which channel tap is significant. In contrast to the significant tap selection approach, the matching pursuit type algorithm is employed in $[7,8]$ to estimate channel taps iteratively by maximizing the correlation of a column of the mixture matrix with the residual signal. More recently, the new field of compressive sensing has proposed effective solutions to exploit channel sparsity, such as $[9,10]$. However, the existing time domain predictors ignore this sparsity of radio channel.

This paper presents a new time domain predictor exploiting the features of sparse channel for OFDM systems. Firstly, the proposed predictor transforms the estimated frequency domain channel samples in time domain and operates on each channel tap. Secondly, the predictor separates the negligible taps from the significant channel taps before performing prediction. The proposed predictor mainly has the following characteristics.

(1) The proposed channel predictor operates on channel taps in the time domain impulse response. Each channel tap contains a number of subpaths, while the variation at each subcarrier of OFDM is a combined variation of all taps. Thus, the single channel tap with less subpaths is more predictable than the subcarrier of OFDM [11].

(2) In sparse channel, many channel taps have no energy at all except for estimation noise. Separating those negligible channel taps from the significant ones 
in channel will eliminate the noise perturbation and make the prediction more accurate.

(3) Since the number of significant channel taps is much smaller than that of OFDM subcarriers, the proposed time domain method operating on the significant channel taps has a much lower prediction complexity than the frequency domain method.

(4) As the number of significant channel taps to be predicted is small, we can employ the predictor with higher complexity but having a superior tracking ability for each tap, without worrying about the overall computational complexity.

The rest of this paper is organized as follows. Section 2 describes the system model. Section 3 proposes the time domain prediction method exploiting the features of sparse channel. Section 4 illustrates the performance evaluation, while Section 5 is the conclusion.

\section{System Model}

We consider an OFDM modulation system with $K$ subcarriers operating over a wideband channel. The receiver architecture is shown in Figure 1. Note that the cyclic-prefix (CP) discarding is omitted in this figure. The received signal is

$$
r[n]=\sum_{l=0}^{L_{t}-1} h[n, l] s[n-l]+z[n],
$$

where $L_{t}$ is the channel's maximum delay, $s[n]$ is the baseband transmit signal, $z[n]$ is additive noise, and $h[n, l]$ is the radio channel given as

$$
h[n, l]=\sum_{i=0}^{L} h\left[n, l_{i}\right] \delta\left[l-l_{i}\right]
$$

where $h\left[n, l_{i}\right]$ is the complex amplitude of $i$ th tap at time $n$, and $L$ is the number of channel taps. Note that $L$ is usually much smaller than $L_{t}$ due to the fact that the time domain impulse response of sparse channel consists of a large number of zero taps in the standard uniform tapped delay model.

In the receiver, the discrete-Fourier-transform (DFT) output frequency domain subcarrier symbol is [3]

$$
R[m ; k]=\operatorname{FFT}_{K}\{r[n]\}=H[m ; k] S[m ; k]+Z[m ; k],
$$

where $R[m ; k]$ is the received signal at the $k$ th subcarrier of the $m$ th OFDM symbol, $S[m ; k]$ is the transmitted signal modulating the $k$ th subcarrier of the $m$ th OFDM symbol, $Z[m ; k]$ is the corresponding noise, and $H[m ; k]$ is the channel coefficient given as

$$
H[m ; k]=\sum_{l=0}^{L_{t}-1} h[m ; l] e^{-j 2 \pi l k / K},
$$

where $h[m ; l]$ is the channel response of the $l$ th delay in the $m$ th OFDM symbol, and it is assumed to be constant over

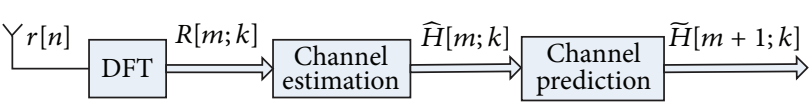

FIGURE 1: OFDM receiver.



FIgURE 2: Time domain predictor for sparse channel.

an OFDM symbol duration. Then, the DFT output $R[m ; k]$ will be processed by the channel estimator, and we have the estimation result as [3]

$$
\widehat{H}[m ; k]=H[m ; k]+\widehat{Z}[m ; k],
$$

where $\widehat{Z}[m ; k]$ is the white Gaussian estimation noise caused by background noise and the receiver imperfections, with variance $\sigma^{2}$. Here, we define the estimation $\mathrm{SNR}$ as $\mathrm{SNR}_{e}=$ $E\left[|H[m ; k]|^{2}\right] / \sigma^{2}$.

Existing channel predictors for OFDM are often realized in the frequency domain. For the $k$ th $(k=0,1, \ldots, K-1)$ subcarrier, the frequency domain method is given by

$$
\widetilde{H}[m+1 ; k]=\sum_{i=0}^{p-1} w_{i}^{*}[m ; k] \widehat{H}[m-i ; k],
$$

where $\widetilde{H}[m+1 ; k]$ is the predicted channel sample at the $k$ th subcarrier, $w_{i}[m ; k]$ is the prediction coefficient at the $k$ th subcarrier, and $p$ is the prediction order. The prediction coefficients can be updated using adaptive algorithm, and it will be introduced in next section.

\section{The Proposed Time Domain Method}

In OFDM systems, the entire channel is divided into many narrow parallel subchannels, and so it is easy to understand why the frequency domain methods realize at each OFDM subcarrier as (6). However, the frequency domain methods will be limited on the following grounds. Firstly, as introduced in Section 1, the subchannel of OFDM is not as predictable as the channel tap in time domain. Secondly, the number of OFDM subcarriers is much larger than that of taps in sparse channel, which leads to a high prediction complexity. Last but not least, the nonsignificant channel taps can be neglected to eliminate the noise perturbation and to make the prediction more accurate. The proposed time domain channel prediction method is described in the following. 


$$
\begin{aligned}
& \begin{array}{l}
\text { For } l=1, \ldots, L_{\mathrm{cp}}-1, \\
\text { Compare }\left|\widehat{\underline{h}}_{1}\right|^{2}, \ldots,\left|\widehat{\underline{h}}_{L_{\mathrm{cp}}}\right|^{2}: \\
\quad \text { (1) Select the } L_{s} \text { significant taps, and perform prediction on each significant tap; } \\
\quad \text { (2) Set the other } L_{\mathrm{cp}}-L_{s} \text { taps as zeros. }
\end{array} \\
& \text { For } l=L_{\mathrm{cp}}, \ldots, K-1, \\
& \quad \text { Set } \widetilde{h}[m+1 ; l]=0 \text { directly. } \\
& \text { (Note: }\left|\widehat{\underline{h}}_{\mid}\right|^{2} \text { is the average power of } l \text { th tap, averaged from the previous estimation samples.) }
\end{aligned}
$$

Algorithm 1: Tap selection algorithm.

3.1. Predictor Architecture. The time domain predictor for sparse channel is shown in Figure 2. The input of the proposed predictor is the frequency domain channel estimation coefficients $\widehat{H}[m ; k], k=0,1, \ldots, K-1$. Firstly, a $K$-point IDFT is applied to the input, which yields

$$
\widehat{h}[m ; l]=\operatorname{IFFT}_{K}\{\widehat{H}[m ; k]\} .
$$

Secondly, tap selection algorithm will select the significant channel taps. Then, if $\widehat{h}[m ; l]$, the $l$ th estimated tap is significant, and it will be applied to the predictor with prediction coefficients $\mathbf{d}[m ; l]$; otherwise, it will be set as zero. Finally, the predicted time domain channel coefficients will be transformed back to frequency domain

$$
\widetilde{H}[m+1 ; k]=\operatorname{FFT}_{K}\{\widetilde{h}[m+1 ; l]\} .
$$

In the proposed method, an additional operation is transformation between time and frequency domains. Due to the employment of FFT implementations, the computational complexity of the transformation is very low. In the following, we introduce the tap selection and the adaptive prediction algorithms.

3.2. Tap Selection. Assume that the CP length of OFDM system is $L_{\mathrm{cp}}$, and the channel's maximum delay does not exceed $L_{\mathrm{cp}}$. Then, for $l=0, \ldots, L_{\mathrm{cp}}-1$, the time domain channel estimation of (7) can be written as

$$
\widehat{h}[m ; l]= \begin{cases}h[m ; l]+\varsigma[m ; l], & \text { significant tap, } \\ \varsigma[m ; l], & \text { zero-valued tap }\end{cases}
$$

while for $l=L_{\mathrm{cp}}, \ldots, K-1$, we have

$$
\widehat{h}[m ; l]=\varsigma[m ; l],
$$

where $\varsigma[m ; l]=\operatorname{IFFT}_{K}\{\widehat{Z}[m ; k]\}$ is the noise, in time domain. In this paper, we use the most significant channel tap approach [5] to identify the significant channel taps. The selection algorithm is shown in Algorithm 1.

3.3. Adaptive Prediction. Since radio channel is time varying in practice, the predictor should adjust itself with the goal of tracking the change of channel. Least-mean-squares (LMS) and recursive-least-squares (RLS) [12] are two major adaptive methods used for channel prediction. It is well known that the LMS predictor is simple to implement, but its ability to track time-varying channel is also limited. In contrast, RLS predictor has a superior tracking ability, but with a much higher complexity. As a result, when computational complexity is a primary concern, the RLS will not be broadly adopted by the frequency domain methods, as too many predictors will be involved in such methods. In this paper, through exploiting the features of sparse channel, the proposed method only uses a small number of predictor. Hence, the RLS can be adopted for channel prediction without worrying about the prediction complexity. For significant channel taps, the RLS predictor is as follows:

$$
\widetilde{h}[m+1 ; l]=\mathbf{d}^{H}[m ; l] \widehat{\mathbf{h}}[m ; l],
$$

where

$$
\begin{gathered}
\mathbf{d}[m ; l]=\left[d_{0}[m ; l], \ldots, d_{p-1}[m ; l]\right]^{T}, \\
\widehat{\mathbf{h}}[m ; l]=[\widehat{h}[m ; l], \ldots, \widehat{h}[m-p+1 ; l]]^{T}
\end{gathered}
$$

are the prediction coefficient vector and the estimated time domain channel coefficient vector, and $p$ is the prediction order. The updated equation of RLS is as follows:

$$
\mathbf{d}[m ; l]=\mathbf{d}[m-1 ; l]+\mathbf{k}[m-1 ; l] e^{*}[m ; l],
$$

where

$$
e[m ; l]=\widehat{h}[m ; l]-\mathbf{d}^{H}[m-1 ; l] \widehat{\mathbf{h}}[m-1 ; l]
$$

is the prediction error, and

$$
\mathbf{k}[m ; l]=\frac{\mathbf{p}[m-1 ; l] \widehat{\mathbf{h}}[m ; l]}{\lambda+\widehat{\mathbf{h}}^{H}[m ; l] \mathbf{p}[m-1 ; l] \widehat{\mathbf{h}}[m ; l]}
$$

is the RLS gain vector, where $\lambda$ is the forgetting factor. Further, the matrix $\mathbf{p}[m ; k]$ can be calculated recursively as

$$
\mathbf{p}[m ; l]=\frac{1}{\lambda}\left(\mathbf{I}-\mathbf{k}[m ; l] \widehat{\mathbf{h}}^{H}[m ; l]\right) \mathbf{p}[m-1 ; l] .
$$

\section{Simulation Results}

In this paper, we compare the NMSE performance of five channel prediction methods. 
(1) Frequency domain (FD) method shows prediction at each OFDM subcarrier in the frequency domain; that is, $\widehat{H}[m ; k], k=0,1, \ldots, K-1$.

(2) Time domain (TD) method shows prediction on each channel tap in the time domain; that is, $\widehat{h}[m ; l], l=$ $0,1, \ldots, K-1$.

(3) The first $L_{\mathrm{cp}}$ taps of time domain $\left(L_{\mathrm{cp}}-T D\right)$ show prediction on each of the first $L_{\mathrm{cp}}$ channel taps; that is, $\widehat{h}[m ; l], l=0,1, \ldots, L_{\mathrm{cp}}-1$.

(4) The $L_{s}$ significant taps of time domain $\left(L_{s}-T D\right)$ show prediction on each of the $L_{s}$ significant channel taps, which are selected by the tap selection algorithm.

(5) Exact time domain (exact-TD) method shows that the $L$ channel taps are known exactly, and the prediction is operated on each of the $L$ channel taps. This method provides a reference in the comparison.

The five methods all employ RLS predictors for prediction. All RLS predictors have the same parameters: the order $p=10$ and the forgetting factor $\lambda=0.9$.

In this paper, we use the standardized 3GPP channel models [13], which model the realistic scattering environments and generate realistic fading datasets, to test our prediction algorithm. The urban microscenario with $L=$ 6 taps is simulated for performance evaluation. The carrier frequency is $2 \mathrm{GHz}$ and the velocity of $\mathrm{MS}$ is $10 \mathrm{~m} / \mathrm{s}$, which leads to a maximum Doppler shift about $f_{\max }=67 \mathrm{~Hz}$. The data sampling rate is set as $8 \mathrm{MHz}$, while the sampling rate used for channel estimation is set as $f_{s}=8 f_{\text {max }}$, which is much lower than the data rate and higher than double of the maximum Doppler shift. In this simulation, 200 channel samples are generated. The first 100 samples are used for observation and the prediction training, while the second 100 samples are for performance evaluation. The FFT size of OFDM system is 128 , the CP length $L_{\mathrm{cp}}$ is 32 , and the number of significant taps is set as $L_{s}=2 L$ according to [5].

The performance of different predictors is compared in terms of the NMSE defined as

$$
\mathrm{NMSE}=\frac{E\left[\|\mathbf{H}(m)-\widetilde{\mathbf{H}}(m)\|^{2}\right]}{E\left[\|\mathbf{H}(m)\|^{2}\right]},
$$

where

$$
\begin{aligned}
\mathbf{H}(m) & =[H[m ; 0], \ldots, H[m ; K-1]]^{T}, \\
\widetilde{\mathbf{H}}(m) & =[\widetilde{H}[m ; 0], \ldots, \widetilde{H}[m ; K-1]]^{T}
\end{aligned}
$$

are real and predicted frequency domain channel coefficient vectors.

The NMSE performance versus the estimation SNR for different predictors is shown in Figure 3. According to Figure 3, we have the following results.

(1) The NMSE performance of TD method is better than that of FD method. It is because the single channel tap is more predictable than the subcarrier of OFDM whose variation is a combined variation of all taps.

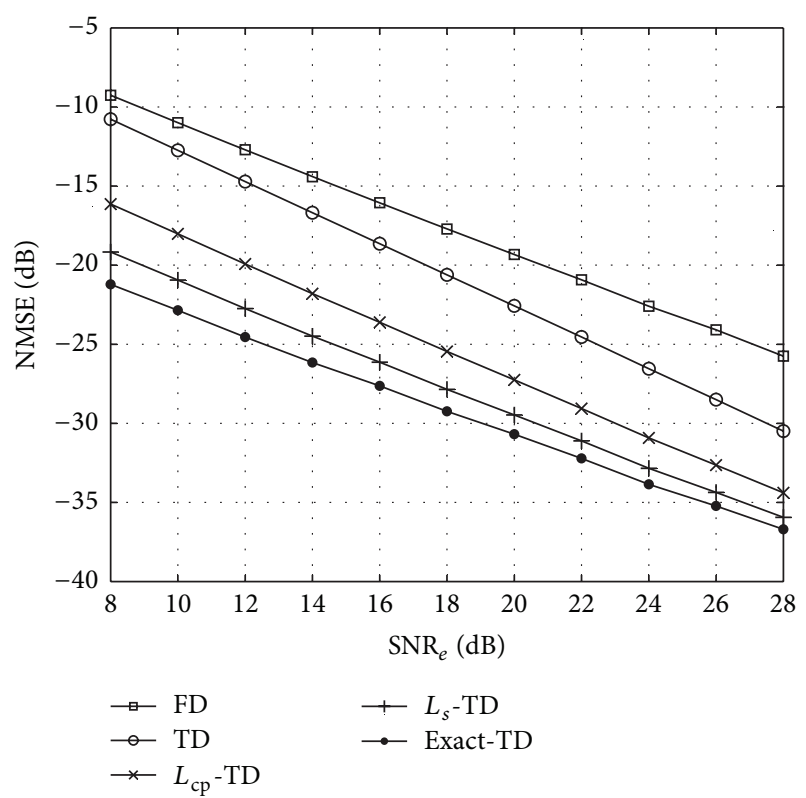

FIGURE 3: NMSE performance comparison for different channel predictors.

(2) The performance of $L_{\mathrm{cp}}-\mathrm{TD}$ is better than TD method. It is because the noise taps whose delays are larger than $L_{\mathrm{cp}}$ are separated.

(3) The performance of $L_{s}$-TD is better than $L_{c p}-T D$. It is because $L_{s}$-TD can separate more negligible taps by exploiting sparse channel features.

(4) The exact-TD method provides a reference in the comparison. It represents the best NMSE performance achievable by exploiting the features of sparse channel.

(5) The numbers of RLS predictors employed by FD, TD, $L_{\mathrm{cp}}-\mathrm{TD}$, and $L_{s}-\mathrm{TD}$ are $K, K, L_{\mathrm{cp}}$, and $L_{s}$, respectively. As $L_{s}$ is small, $L_{s}$-TD has a lower computational complexity than the other three methods.

\section{Conclusion}

A new time domain approach for OFDM channel prediction is proposed, based on the features of sparse channel that many channel taps have no energy at all except for estimation noise, as well as the fact that the channel tap in time domain is more predictable than the subchannel in frequency domain. The proposed method separates the negligible channel taps and only performs prediction on the significant ones. Also, the RLS algorithm with superior tracking ability is employed to predict each significant tap. Although the coefficients update of RLS is complex, the overall computational complexity of the prediction method is not high, due to the limited quantity of significant channel taps. The effectiveness of the proposed approach is validated by the simulations on the physical channel model. As a result, the novel channel 
prediction method can exploit the features of sparse channel and improve the prediction performance.

Future works will focus on the application of the proposed approach to adaptive transmission schemes in multiple antenna systems, including transmit selection diversity and adaptive beamforming.

\section{References}

[1] L. J. Cimini Jr., "Analysis and simulation of a digital mobile channel using orthogonal frequency division multiplexing," IEEE Transactions on Communications, vol. 33, no. 7, pp. 665675, 1985.

[2] M. Sternad, T. Svensson, T. Ottosson, A. Ahlen, A. Svensson, and A. Brunstrom, "Towards systems beyond 3G based on adaptive OFDMA transmission," Proceedings of the IEEE, vol. 95, no. 12, pp. 2432-2455, 2007.

[3] A. Duel-Hallen, H. Hallen, and T. S. Yang, "Long range prediction and reduced feedback for mobile radio adaptive OFDM systems," IEEE Transactions on Wireless Communications, vol. 5, no. 10, pp. 2723-2732, 2006.

[4] D. Schafhuber and G. Matz, "MMSE and adaptive prediction of time-varying channels for OFDM systems," IEEE Transactions on Wireless Communications, vol. 4, no. 2, pp. 593-602, 2005.

[5] H. Minn and V. K. Bhargava, "An investigation into timedomain approach for OFDM channel estimation," IEEE Transactions on Broadcasting, vol. 46, no. 4, pp. 240-248, 2000.

[6] C. Carbonelli, S. Vedantam, and U. Mitra, "Sparse channel estimation with zero tap detection," IEEE Transactions on Wireless Communications, vol. 6, no. 5, pp. 1743-1753, 2007.

[7] S. Kim, "Angle-domain frequency-selective sparse channel estimation for underwater MIMO-OFDM systems," IEEE Communications Letters, vol. 16, no. 5, pp. 685-687, 2012.

[8] X. Jiang, W.-J. Zeng, and E. Cheng, "A fast algorithm for sparse channel estimation via orthogonal matching pursuit," in Proceedings of the 73rd Vehicular Technology Conference (VTC Spring), pp. 1-5, May 2011.

[9] C. R. Berger, Z. Wang, J. Huang, and S. Zhou, "Application of compressive sensing to sparse channel estimation," IEEE Communications Magazine, vol. 48, no. 11, pp. 164-174, 2010.

[10] W. U. Bajwa, J. Haupt, A. M. Sayeed, and R. Nowak, "Compressed channel sensing: a new approach to estimating sparse multipath channels," Proceedings of the IEEE, vol. 98, no. 6, pp. 1058-1076, 2010.

[11] T. Eyceoz, S. Hu, and A. Duel-Hallen, "Performance analysis of long range prediction for fast fading channels," in Proceedings of the 33rd Annual Conference on Information Sciences and Systems (CISS '99), vol. 2, pp. 656-661, 1999.

[12] S. Haykin, Adaptive Filter Theory, Prentice Hall, 3rd edition, 2002.

[13] "Spatial channel model for multiple input multiple output (MIMO) simulations," 3GPP Technical Report 25. 996 V6. 1. 0, Chichester, UK, 2003, http://www.3gpp.org/ftp/Specs/htmlinfo/25996.htm. 

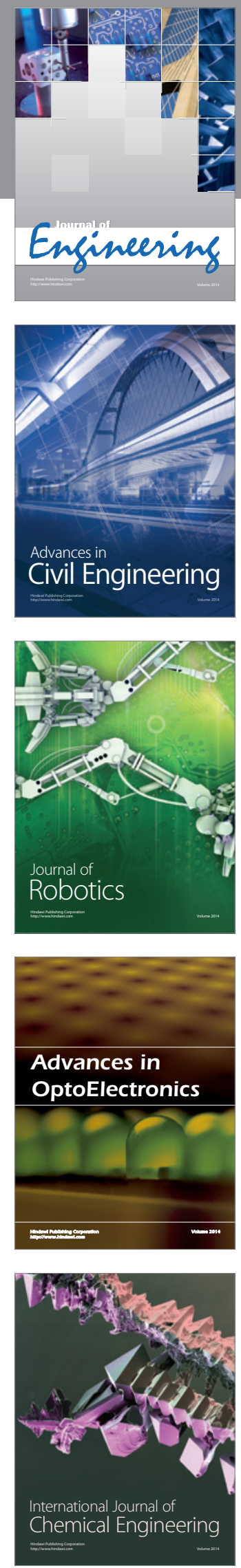



The Scientific World Journal


Submit your manuscripts at http://www.hindawi.com
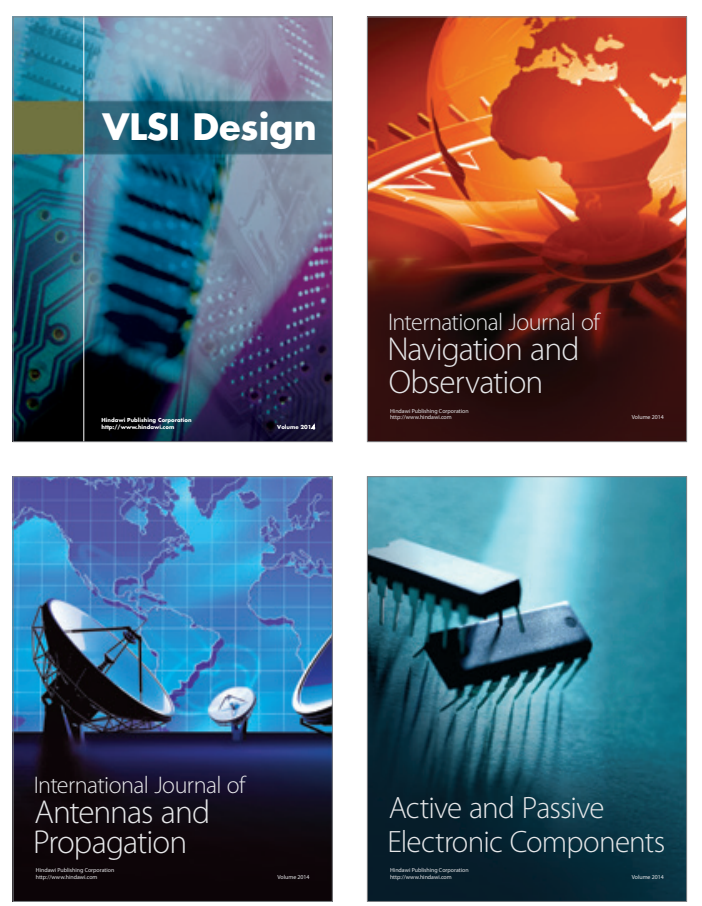
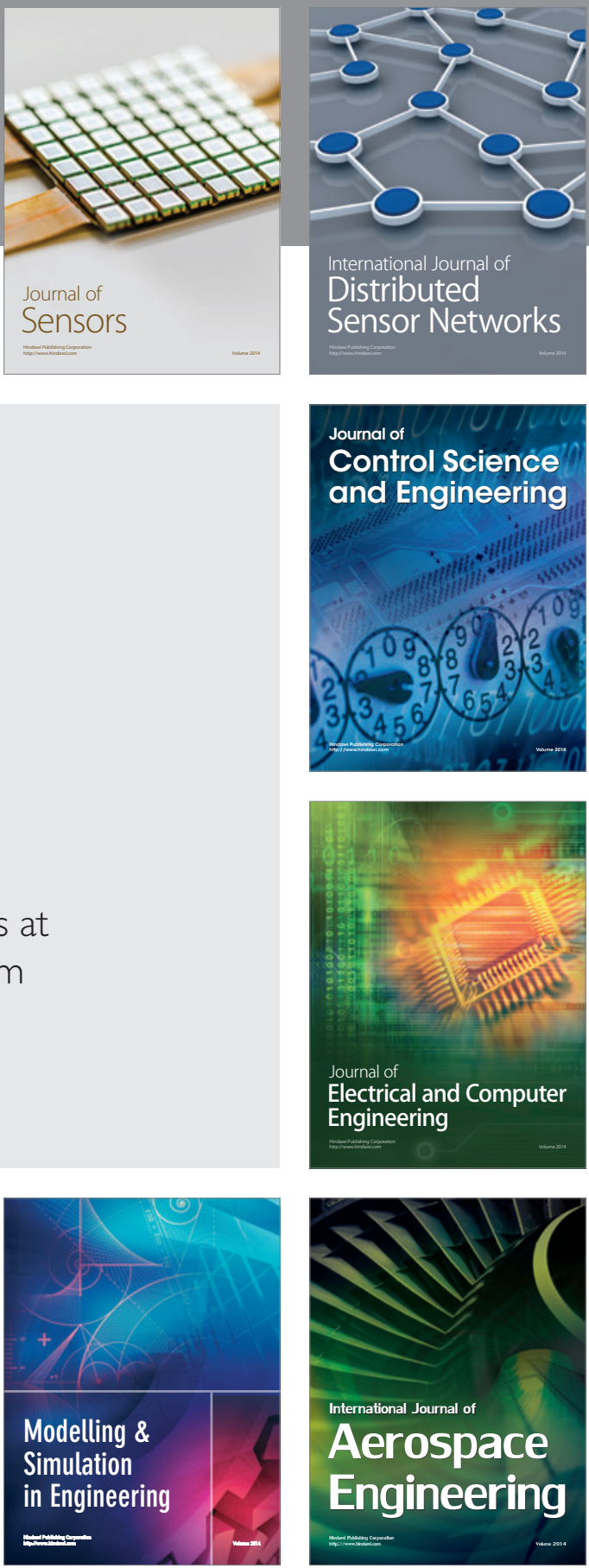

Journal of

Control Science

and Engineering
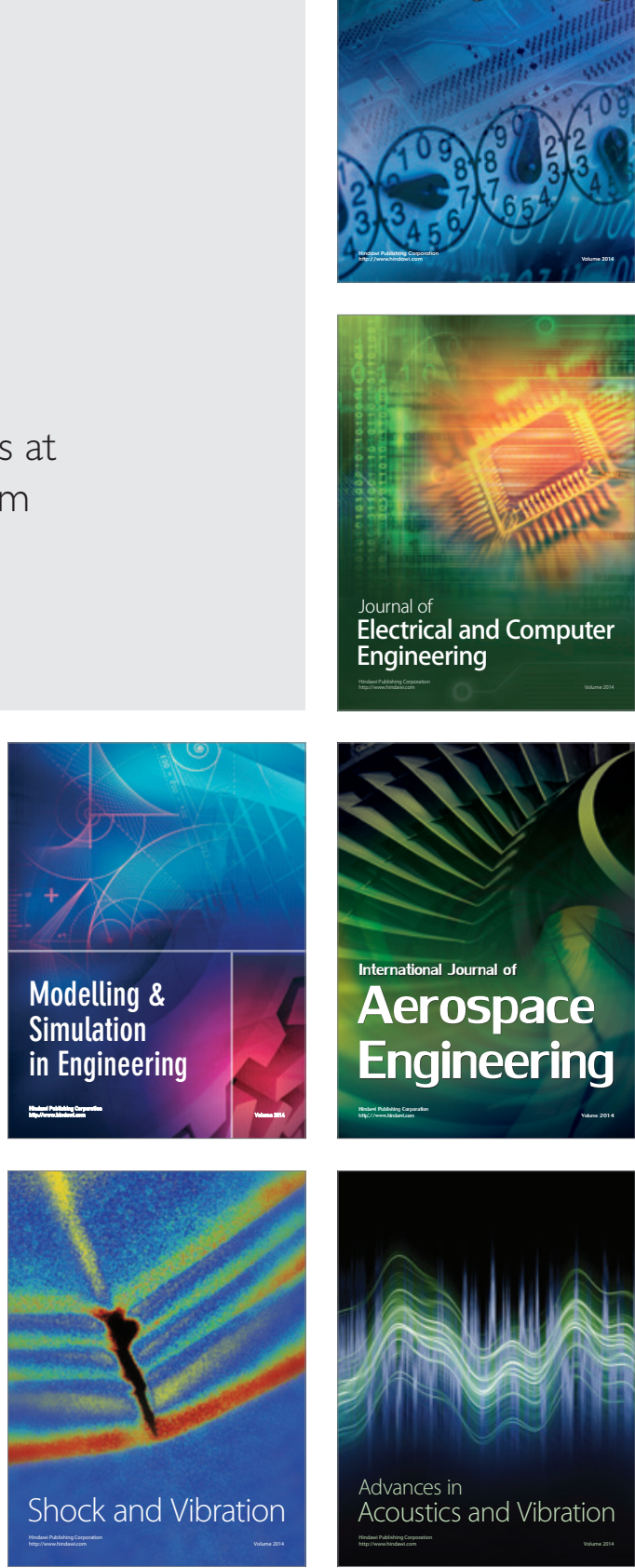